\title{
ENSAMBLAJE DE HORMIGAS DEL BOSQUE SECO TROPICAL, JARDIN BOTÁNICO DE CALI
}

\author{
Assembly Ants Tropical Dry Forest, Cali Botanicall Garden \\ María Cristina Gallego-Ropero¹ \& Beatriz Salguero Rivera ${ }^{2}$
}

Gallego-Ropero, M.C. \& Salguero, B. (2015). Ensamblaje de hormigas del bosque seco tropical, jardín botánico de Cali. Colombia Forestal, 18(1), 139-150

Recepción: 26 de julio de 2014

\section{RESUMEN}

Se presentan los resultados acerca del ensamblaje de hormigas de un fragmento de Bosque Seco Tropical secundario localizado en el Jardín Botánico de Cali. Se escogieron dos hábitats: el fragmento de bosque y su matriz de potrero. Se realizaron muestreos mensuales por seis meses, usando un transecto lineal de 100 m, con 10 estaciones distanciadas 10 m. En cada estación se aplicaron diferentes técnicas de muestreo: trampas pitfall; cebo epígeo y arbóreo; cernido y extracción de $1 \mathrm{~m}^{2}$ de hojarasca y captura manual. Se determinó la riqueza, abundancia y diversidad de hormigas por hábitat. Se calcularon los coeficientes de correlación y de regresión entre la temperatura, la humedad relativa y la riqueza de hormigas. Se colectó un total de 13170 hormigas, agrupadas en 55 especies, 35 géneros y seis subfamilias. El bosque presentó la mayor riqueza de especies con el $90.9 \%$. Esta riqueza se constituye en una valiosa contribución a la conservación de la mirmecofauna del Valle del Cauca. A pesar de que el bosque se encuentra en un proceso de regeneración y que su matriz está fuertemente perturbada por constantes incendios e intervención humana, la riqueza de especies de hormigas muestra que este es una fuente de recursos biológicos muy valiosa para la conservación de las especies.
Aprobación: 20 de noviembre de 2014

Palabras clave: ecosistemas secos, Formicidae, fragmentación, Hymenoptera.

\begin{abstract}
The ant assemblages of a fragment of secondary tropical dry forest located in the Botanical Garden of Cali are presented. Two habitats were chosen: a fragment of forest and a pasture matrix. Samples were taken monthly for six months, using a linear transect of 100 $\mathrm{m}$, with 10 stations spaced $10 \mathrm{~m}$. At each station pitfaII traps, epigeal bait and arboreal, sifting and removal of $1 \mathrm{~m}^{2}$ of litter and manual capture survey techniques were applied. Richness, abundance and diversity of ant habitats were determined. Correlation coefficients and regression between temperature, relative humidity and richness of ants were calculated. A total of 13 170 ants representing 55 species, 35 genera and six subfamilies were collected. The forest had the highest species richness with $90.9 \%$. This wealth of diversity indicates the conservation value of the Valle del Cauca myrmecofauna. Although the forest is in the process of regeneration, strongly disturbed by constant fire and human intervention, the ant species richness shows that it remains an invaluable source of biological resources for the conservation of species.
\end{abstract}

Keys words: dry ecosystems, formicidae, fragmentation, hymenoptera.

Departamento de Biología, Universidad del Cauca. Cali, Colombia. mgallego@unicauca.edu.co. Autor para correspondencia. Facultad de Ciencias Básicas, Universidad Autónoma de Occidente. Cali, Colombia. bsalguero@uao.edu.co 


\section{INTRODUCCIÓN}

En Colombia los esfuerzos de conservación se han situado en un lugar prioritario a escala global debido a su gran riqueza biológica (McNelly et al., 1990) y en la última década se ha presentado un aumento en la deforestación, transformación de hábitats naturales y sobre-explotación. Desde 1950 hasta 1986 las áreas cubiertas por pastos aumentaron de 12.1 a 26.7 millones de hectáreas y actualmente es utilizado el $73.3 \%$ del territorio nacional para actividades agrícolas y ganaderas $(\mathrm{IAvH}, 1998)$.

Las hormigas son considerada excelentes indicadoras del estado de conservación de los bosques (Kremen, 1994) y han recibido principal atención en los departamentos del Valle del Cauca, Chocó y Bolívar en estudios realizados en bosques tropicales secos y húmedos (Armbrecht, 1995; 1996; 1999; Armbrecht \& Chacón de Ulloa, 1999) y han reflejado gracias a sus resultados la importancia de dichos fragmentos en la conservación de la diversidad de especies. A fin de establecer especies promisorias indicadoras de la calidad de hábitat en estos bosques, trabajos similares han comparado la mirmecofauna de fragmentos de bosque con sus matrices, además han permitido sustentar su importancia biológica.

Estudios de investigación relacionados con la comparación de la riqueza en fragmentos de bosque, con respecto a sus matrices antropogénicas, han arrojado resultados similares, como los realizados por Armbrecht \& Chacón de Ulloa (1999); en los cuales se encontró que la riqueza en los fragmentos de bosque es mayor que sus matrices debido a que esta se encuentra asociada a la gran diversidad de especies de plantas del fragmento. Por otro lado, Roth et al. (1994) ejecutaron investigaciones en Costa Rica y encontraron una mayor diversidad en el suelo del bosque primario y un cacaotal abandonado en comparación con plantaciones de banano y cacao.

En contraste, la diversidad de hormigas en bosques fragmentados y sus matrices antropogénicas han mostrado resultados visibles sobre el número de especies exclusivas de tales relictos, lo que demuestra que los bosques más ricos en estas especies preservan una mayor diversidad regional de ejemplares raros (Armbrecht \& Chacón de Ulloa, 1999), al igual que las investigaciones realizadas en bosques húmedos en el bajo Calima (Aldana \& Chacón de Ulloa, 1995) y en bosque nublado en los Farallones de Cali (Bustos \& Chacón de Ulloa, 1996).

Al tener en cuenta la posible cualidad de las hormigas como indicadoras de la calidad de ecosistemas (Roth et al,. 1994; Buckley, 1982; Beattie, 1985; Davidson \& Mckey, 1993), el presente estudio tuvo como objetivo determinar cómo cambia la comunidad de hormigas en el fragmento de bosque del Jardín Botánico de Cali y su matriz de pastizal, el cual conserva uno de los fragmentos de bosque seco tropical del Valle del Cauca que ha sido poco estudiado; y a partir de allí, con la información obtenida, se puedan proponer estrategias de conservación que permitan mejorar el estado sucesional en el Jardín Botánico de Cali.

\section{MATERIALES Y MÉTODOS}

\section{Área de estudio}

El estudio se llevó a cabo en el Jardín Botánico de Cali ubicado a $3^{\circ} 27^{\prime} 11.3^{\prime \prime} \mathrm{N}$ y $76^{\circ} 34^{\prime} 24.1^{\prime \prime} \mathrm{W}$ de la vertiente izquierda del río Cali entre las vertientes de los ríos Cali y Aguacatal, que se sitúa dentro de la Comuna 1 y el corregimiento de Los Andes (Orjuela, 2004). La zona boscosa del Jardín se ha establecido a lo largo de 80 años, en un área de doce hectáreas (Orejuela \& Contreras, 2006). Según Holdridge (Espinal, 1967), se clasifica como bosque seco tropical (bs-T). Presenta un rango de temperatura promedio que varía de $26^{\circ}$ a $28^{\circ} \mathrm{C}$, una precipitación anual entre los 600 y 700 mm y entre 1100 y 1125 m de altitud. La precipitación presenta una distribución de tipo bimodal con dos periodos lluviosos de marzo a mayo y de septiembre a noviembre, en el que julio es el mes de 
menor precipitación (CVC, 2000). En el Jardín Botánico de Cali se destacan por lo menos seis especies de los estratos altos como Arrayán (Myrcia popayanensis), Laurel jigua (Cynammomum triplinerve), Sangregao (Croton gossypifolius), Guácimo (Guazuma ulmifolia), Chiminango (Pithecellobium dulce) y Chagualo (Clusia sp.). Las plantas del estrato medio son Sangregao (Croton sp.), Arrayán (Myrcia), Guayaba (Psidum guajava), Verraquillo (Tremamicrantha), Carbonero (Calliandra pittieri), Jigua (Cynammomum), Guácimo (Guazuma pertenece a ambos estratos), Leucaena (Leucaena leucocephala), Chagualo (Clusia sp.), (Solanum sp.) y Miconia. En el estrato bajo sobresalen Cordoncillo (Piper sp.) y Anamú (Petiveria alliacea) de la familia Phytolacaceae. El bosque presenta abundancia de plantas trepadoras y de bejucos, dominando especies de las familias Aristolochiaceae, Passifloraceae y Cucurbitaceae (Orejuela \& Contreras, 2006). Dentro del paisaje en el cual se encuentra inmerso el Jardín Botánico, se encuentra un antiguo potrero de aproximadamente media hectárea que se caracteriza por poseer una alta pendiente y vegetación muy pobre, la cual está constituida por pastizales en su totalidad. La zona es sometida a una constante perturbación urbana y a quemas frecuentes y fue tomada como el hábitat dos para los muestreos.

\section{Métodos de muestreo}

En cada hábitat seleccionado, bosque y pastizal, se trazó un transecto de $100 \mathrm{~m}$ y en cada uno se ubicaron 10 puntos de muestreo separados $10 \mathrm{~m}$ entre sí. El primer punto se instaló a 20 m desde el borde. Se realizaron cinco tipos de muestreo por estación en el bosque y cuatro en el potrero, con el propósito de obtener una muestra representativa de las especies presentes en el fragmento de bosque y el potrero: una trampa de caída (pitfall) en el suelo (modificada de Jaffé et al., 1993), las trampas se dejaron actuar por un periodo de cinco a seis horas, donde se colectaron las hormigas atrapadas. Este método de muestreo es objetivo y confiable y permite capturar hormigas que están activas en la superficie del suelo (Bestelmeyer et al., 2000), inclusive las hormigas crípticas (Samways, 1983). Se colocó un cebo de atún epigeo dispuesto sobre el suelo y otro hipogeo (en bosque), el cebo se elaboró con un trozo de papel de aproximadamente $10 \times 15 \mathrm{~cm}$ con 3 g de atún en aceite, estos se dejaron actuar durante dos horas. En cada estación se trazó un cuadrante de $1 \mathrm{~m}^{2}$ donde se realizó durante 10 minutos una búsqueda visual y captura manual en diferentes sustratos (troncos en descomposición, ramas secas, suelo, vegetación, bajo piedras y pistas de forrajeo). Por último, en cada estación se recogió la hojarasca presente en $1 \mathrm{~m}^{2}$ y se pasó por un saco cernidor tipo mini-Winkler (Jaffé et al., 1993). El material vegetal cernido se dejó 48 horas en los sacos mini-Winkler y luego se separaron y rotularon las muestras. Las variables ambientales de temperatura y humedad relativa fueron medidas. Se realizó un muestreo por mes durante seis meses, tanto en el fragmento de bosque como en el potrero para cubrir un ciclo de lluvias, uno de transición y un periodo de verano y así asegurar una buena colecta de ejemplares. El material colectado se guardó en alcohol al $70 \%$. Las muestras fueron Ilevadas al Laboratorio de Ciencias Ambientales de la Universidad Autónoma de Occidente donde se procedió a limpiar, montar e identificar los ejemplares. Para la determinación taxonómica se utilizaron las claves de Holldobler \& Wilson (1990), Boltón (1994) y Palacio \& Fernández (2003).

\section{Análisis de datos}

Con el propósito de evaluar la diversidad local $\alpha$ en bosque y pastizal, se determinó la abundancia de hormigas con base en el número de individuos para cada especie y la riqueza como el número de especies en cada sitio de muestreo. Se calculó el índice de diversidad de Shannon $\left(\mathrm{H}^{\prime}\right)$ y mediante el análisis de curvas de acumulación de especies se evaluó la eficiencia de los muestreos usando el programa EstimateS 5.1 (Colwell, 1998). Para eliminar el efecto de las diferentes técnicas de colecta 
utilizadas en el estudio, así como el orden en el cual se adicionaron las muestras, se aleatorizaron 100 veces. Se emplearon estimadores basados en la incidencia (presencia-ausencia) por ser más apropiados en el análisis de grupos hiperdiversos y con distribuciones agrupadas (Longino, 1994), como Chao 2 y Jacknife 2. A los datos obtenidos de riqueza de especies para cada hábitat, se les aplicó un análisis de varianza de dos factores (SPSS, versión 13), teniendo en cuenta para cada hábitat las variables época de muestreo y tipo de muestreo, además de la interacción entre estas. Como variable dependiente se designó la riqueza de especies de hormigas. Con el fin de determinar si existe una relación entre la comunidad de hormigas y las variables ambientales medidas, se obtuvieron los coeficientes de correlación y de regresión entre estas variables y la riqueza de hormigas.

\section{RESULTADOS}

\section{Composición general de la mirmecofauna}

Se colectó un total de 13170 hormigas, agrupadas en 55 especies. Las especies encontradas se pueden separar taxonómicamente en 35 géneros, pertenecientes a seis subfamilias, que son en orden de riqueza Myrmicinae, Ponerinae, Formicinae, Dolichoderinae, Pseudomyrmecinae y Dorylinae. Dicha cifra corresponde al 39\% de los géneros y al $6.05 \%$ de las especies registradas para Colombia (Fernández, 2003; Fernández \& Sendoya, 2004; Chacón de Ulloa \& Armbrecht, 2006).

La distribución de las especies en cada género y de los géneros en cada una de las subfamilias, se relacionan en la Tabla 1, así como el número de géneros para catorce fragmentos de bosque seco de la cuenca media del río Cauca reportados por Chacón de Ulloa \& Armbrecht (2006), en estudios realizados sobre la caracterización de la mirmecofauna de fragmentos de bosque seco y sus matrices.

En el fragmento de bosque se presenta una estructura arbórea más compleja y se registró un mayor número de especies $90.9 \%$ del total colectado, a diferencia del potrero ralo, ecosistema menos complejo y más simplificado que presentó una menor riqueza de especies 60.1\% (Figura 1).

De las 55 especies encontradas, 50 se capturaron al menos una vez en bosque y 38 al menos una vez en potrero, la riqueza de especies registrada difiere significativamente entre los dos

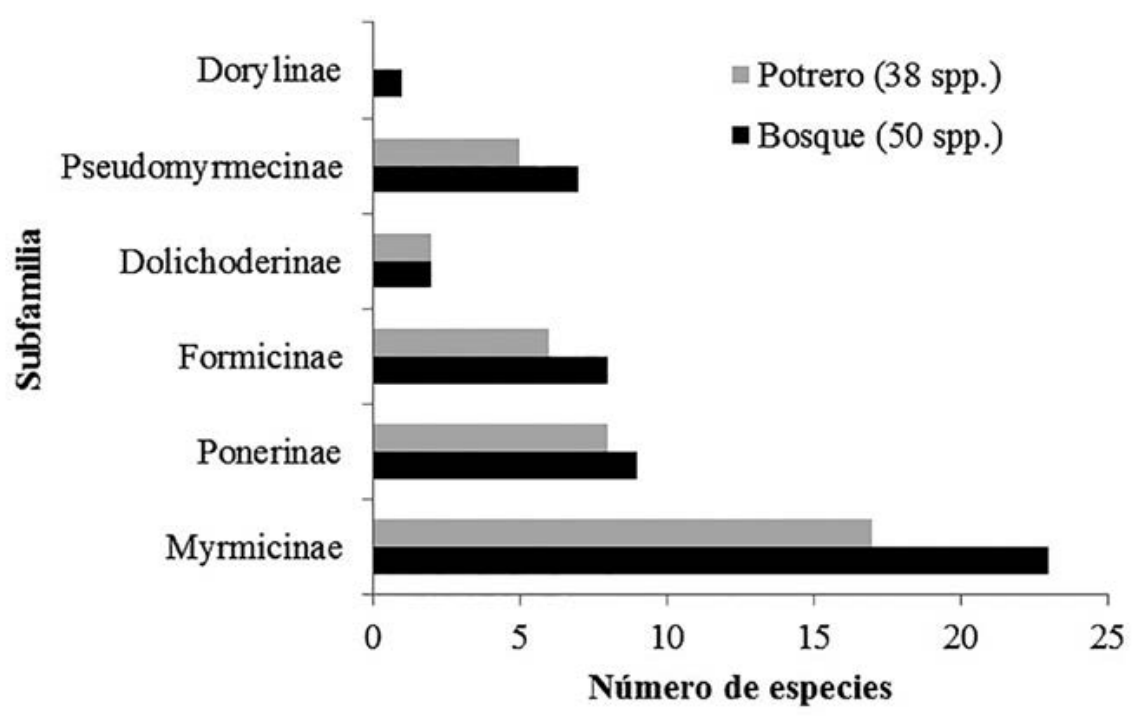

Figura 1. Riqueza de especies por subfamilia colectadas en los dos hábitats: bosque seco tropical y potrero. 
Tabla 1. Número de especies por género y por cada una de las seis subfamilias encontradas en los dos hábitats: bosque seco y potrero.

\begin{tabular}{|c|c|c|c|c|}
\hline Subfamilia & Género & $\begin{array}{l}\text { Número de } \\
\text { géneros } \\
\text { JBC }\end{array}$ & $\begin{array}{l}\text { Número de } \\
\text { especies por } \\
\text { género JBC }\end{array}$ & $\begin{array}{l}\text { Número de géneros } \\
\text { en } 14 \text { relictos de } \\
\text { bosque seco* }\end{array}$ \\
\hline \multirow[t]{19}{*}{ Myrmicinae } & Acanthognatus & 19 & 1 & 27 \\
\hline & Atta cephalotes (Linneus 1758) & & 1 & \\
\hline & Cephalotes cf. maculatus (Smith 1876) & & 1 & \\
\hline & Crematogaster & & 1 & \\
\hline & Cyphomyrmex & & 1 & \\
\hline & Lepthototrax & & 1 & \\
\hline & Megalonomyrmex & & 1 & \\
\hline & Monomorium & & 1 & \\
\hline & Myсосерurus & & 1 & \\
\hline & Octostruma & & 1 & \\
\hline & Pheidole & & 4 & \\
\hline & Procryptocerus & & 1 & \\
\hline & Pyramica & & 1 & \\
\hline & Rogeria & & 1 & \\
\hline & Solenopsis & & 2 & \\
\hline & Strumigenys & & 1 & \\
\hline & Tetramorium & & 1 & \\
\hline & Trachymyrmex & & 1 & \\
\hline & Wasmannia auropunctata (Roger 1863) & & 1 & \\
\hline \multirow[t]{6}{*}{ Ponerinae } & Anochetus & 6 & 1 & 12 \\
\hline & Ectatoma ruidum (Roger 1861) & & 1 & \\
\hline & Gnamptogenys & & 1 & \\
\hline & Hypoponera & & 3 & \\
\hline & Odontomachus & & 1 & \\
\hline & Pachycondyla harpax (Fabricius 1804) & & 3 & \\
\hline \multirow[t]{4}{*}{ Formicinae } & Acropyga & 4 & 2 & 6 \\
\hline & Brachymyrmex & & 2 & \\
\hline & Camponotus & & 3 & \\
\hline & Nylanderia & & 2 & \\
\hline \multirow[t]{4}{*}{ Dolichoderinae } & Azteca & 4 & 1 & 4 \\
\hline & Dolichoderus & & 1 & \\
\hline & Dorymyrmex & & 1 & \\
\hline & Linepithema neotropicum (Wild, 2007) & & 1 & \\
\hline Pseudomyrmecinae & Pseudomyrmex & 1 & 8 & 1 \\
\hline Dorylinae & Neivamyrmex & 1 & 1 & 4 \\
\hline Total & & 35 & 55 & 54 \\
\hline
\end{tabular}

*Chacón de Ulloa \& Armbrecht, 2006 
hábitats (F: 92.011; p: 0.001). Del total de especies, 33 fueron colectadas al menos una vez en ambos sitios. De las 50 especies presentes en el bosque, la subfamilia más representativa fue Myrmicinae con veintitrés morfoespecies que corresponden al $41.8 \%$ del total para el bosque; la subfamilia con menos representantes fue Dorylinae, que registró una sola especie, correspondiendo al $1.8 \%$ del total encontrado. De las 38 especies muestreadas en la matriz de potrero, la subfamilia Myrmicinae presentó el mayor número de especies con 17 de ellas que corresponden al $30.9 \%$ del total para el potrero. La subfamilia con menor número de especies fue Dolichoderinae, con dos especies que corresponden al 3.6\%
(Tabla 2). La subfamilia Dorylinae no tuvo ningún representante en el potrero.

En cuanto a la abundancia, en el bosque se colectó el 70.79\% (9324) de todos los individuos (13 170) y en el potrero el $29.20 \%$ (3846). En la figura 2 se observa el porcentaje de individuos colectados por subfamilias para cada hábitat.

\section{Diversidad de especies y estimadores de riqueza}

En la curva de saturación de especies para el bosque se observa la tendencia a una asíntota en el valor 50 (especies), los estimadores predicen un aumento de casi dos especies, lo que permitió

Tabla 2. Proporción de géneros encontrados por cada subfamilia en los dos hábitats: bosque seco tropical y potrero.

\begin{tabular}{lcc}
\hline \multicolumn{1}{c}{ SUBFAMILIA } & BOSQUE (32) & POTRERO (24) \\
\hline Myrmicinae & $41.8(19)$ & $30.9(13)$ \\
Ponerinae & $16.4(5)$ & $14.5(5)$ \\
Formicinae & $14.5(4)$ & $10.9(3)$ \\
Dolichoderinae & $3.6(2)$ & $3.6(2)$ \\
Pseudomyrmecinae & $12.7(1)$ & $9.1(1)$ \\
Dorylinae & $1.8(1)$ & \\
\hline
\end{tabular}

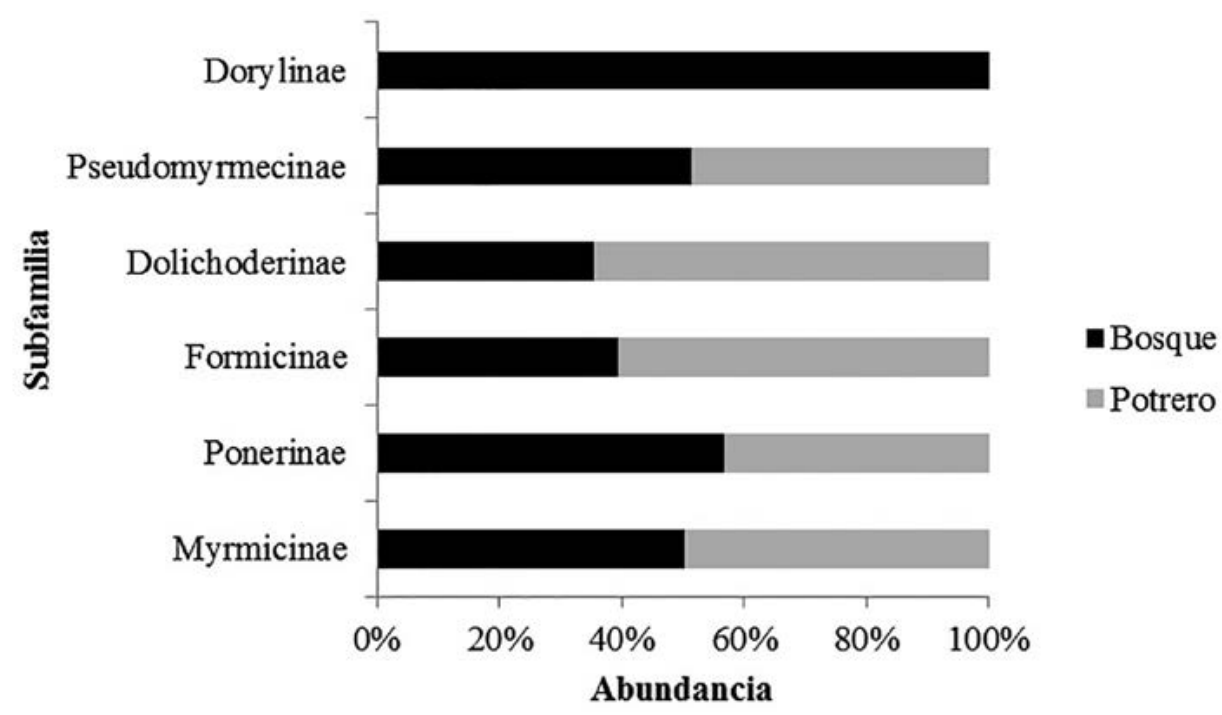

Figura 2. Porcentaje de hormigas colectadas por subfamilias en cada hábitat: bosque seco tropical y potrero. 
calcular la eficiencia de muestreo en un 97.23\%. Para el potrero ocurrió algo similar, la curva presenta una asíntota en el valor 38 (especies), los estimadores predicen un aumento de casi dos especies, presentando una eficiencia de muestreo del 96.74\% (Figura 3; Tabla 3).

Los valores de los estimadores Chao 2, Jacknife 2 y el número de especies observadas para cada uno de los hábitats (diversidad local) y la riqueza total (regional) se visualizan en la tabla 3. A nivel regional se observaron 55 especies, la estimación de la diversidad esperada para esta región con el estimador Jacknife2, predice que aproximadamente tres especies adicionales deberían haber sido encontradas en el estudio (Chao $2 \pm 2.19$
$\mathrm{SD})$. De lo anterior se deduce que según este estimador, el $94.7 \%$ de la diversidad esperada para la región fue registrada en el muestreo.

A nivel general (bosque y potrero) el valor calculado del Índice de Shannon fue de 3.26, el cual es un valor relativamente alto, lo cual sugiere que a este nivel la diversidad de especies de hormigas es moderadamente alta. Si observamos cada hábitat por separado, en el caso del bosque el valor del índice es mucho mayor que para el potrero, siendo diferentes significativamente ( $p$ : 0.001). Al calcular el índice de equitabilidad se observa para el bosque un valor de 0.683 y un valor para el índice de dominancia de 0.112 (Tabla 3) (Moreno, 2001). Para el potrero ocurre algo diferente, el

Tabla 3. Valores observados y estimados de riqueza y diversidad de especies de hormigas en los dos hábitats: bosque seco tropical y potrero.

\begin{tabular}{lcccc}
\hline \multicolumn{1}{c}{ Hábitat } & Bosque & Potrero & $\begin{array}{c}\text { Diversidad } \\
\text { regional }\end{array}$ & $\begin{array}{c}\text { Índice de } \\
\text { complementariedad }\end{array}$ \\
\hline Especies observadas & 50 & 38 & 55 & 0.4 \\
Chao 2 & 51.04 & 39.06 & 57.02 & \\
Jacknife 2 & 51.42 & 39.28 & 56.98 & \\
Eficiencia de muestreo (\%) & 97.23 & 96.74 & 94.70 & \\
Índice de Diversidad Shannon & 2.673 & 1.855 & 3.26 & \\
Índice de Dominancia & 0.112 & 0.304 & & \\
Índice de Equitabilidad & 0.683 & 0.509 & & \\
\hline
\end{tabular}

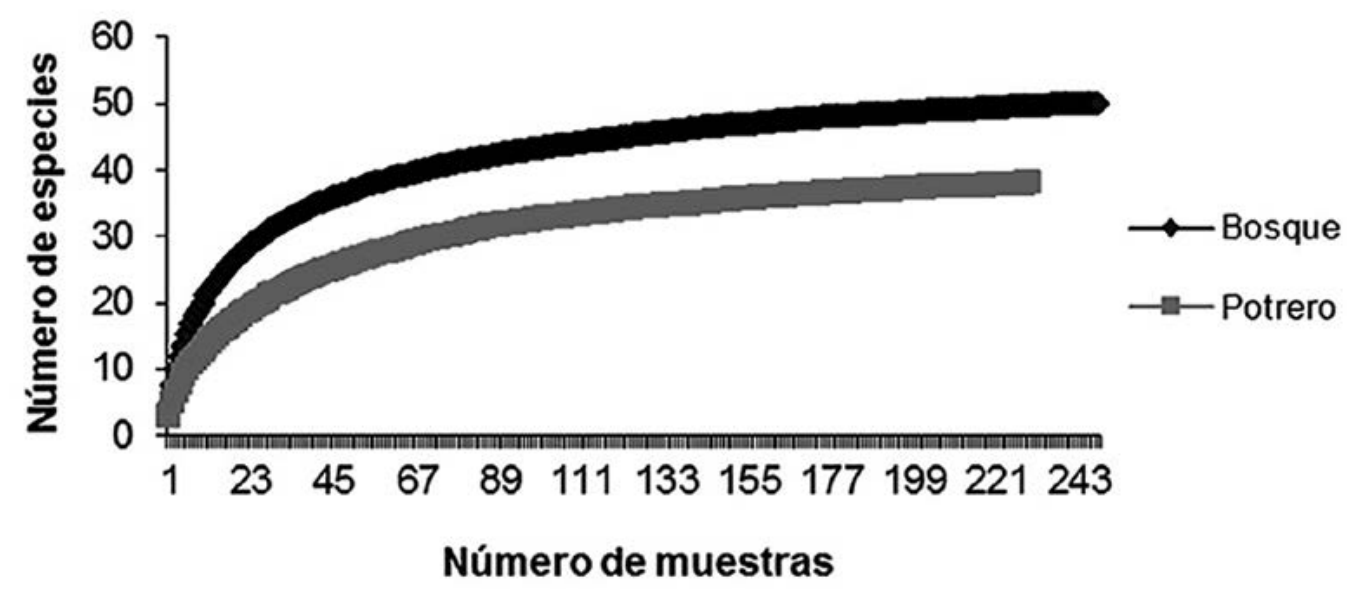

Figura 3. Curva de acumulación de especies para cada hábitat: bosque seco tropical y potrero. 
valor del índice de dominancia de 0.304 es mayor que el presentado en el bosque, a su vez el valor del índice de equitabilidad de 0.509 es menor que el del bosque. El valor calculado del índice de complementariedad entre el bosque y el potrero fue de 0.4 , lo cual indica que los dos hábitats estudiados difieren en un $40 \%$, por lo tanto se complementan en un $60 \%$ de las especies (Colwell \& Coddington, 1994). De las 55 especies de hormigas encontradas, se reportan 17 especies exclusivas para el bosque y cinco especies exclusivas para el potrero de los géneros Dorymyrmex, Linepithema, Odontomachus y Pseudomyrmex.

\section{Variables ambientales versus abundancia y riqueza de hormigas}

\section{Temperatura ambiente promedio}

El rango de la temperatura ambiente promedio durante los seis meses de muestreo para los hábitats evaluados osciló entre $23.4^{\circ} \mathrm{C}$ y $36.2^{\circ} \mathrm{C}$. Para el bosque el coeficiente de correlación calculado de -0.36 para riqueza y -0.49 para abundancia, sugieren que el aumento o reducción en la abundancia y riqueza de hormigas presentó una relación inversa con esta variable ambiental; es decir, en la medida que la temperatura aumentó, bajo las condiciones del bosque, disminuyó la riqueza y abundancia de la comunidad de hormigas. Contrariamente, en el potrero se presentaron valores positivos de los coeficientes de correlación calculados, para riqueza fue de 0.89 y para abundancia de 0.76; lo que indica que en el caso de este hábitat en la medida en que aumenta la temperatura tanto la riqueza como la abundancia de hormigas aumenta, esta tendencia curiosamente se puede explicar al observar que se presentan especies de hormigas típicas de estas condiciones ambientales.

\section{Humedad relativa}

El rango de humedad relativa de los hábitats evaluados estuvo entre 41.5 y $93.4 \%$. Para el bosque los coeficientes de correlación calculados, tanto para la abundancia (0.73) como para la riqueza (0.44), fueron positivos, sugiriendo que esta variable puede estar influenciando directamente la riqueza y abundancia de hormigas; es decir una mayor cobertura vegetal permite retener mayor cantidad de vapor de agua en el ambiente que se ve reflejado incluso en una disminución de la temperatura ambiental.

En el caso del potrero, los coeficientes de correlación calculados, tanto para la abundancia -0.54 como para la riqueza -0.70 , fueron negativos, es decir la relación es inversa. Como la temperatura en este hábitat es muy alta y no hay cobertura vegetal, no se presenta un ambiente tan húmedo como en el bosque.

\section{Análisis de varianza}

Para el bosque se presentan diferencias significativas en la variable tipo de muestreo y la interacción época de muestreo-tipo de muestreo, pero no se presentan diferencias entre las épocas de muestreo que hacen referencia a los diferentes meses en los cuales se llevó a cabo el estudio (Tabla 4).

Tabla 4. Análisis de varianza multifactorial.

\begin{tabular}{clccc}
\hline Hábitat & \multicolumn{1}{c}{ Variable } & gl & F & p \\
\hline Bosque & Época de muestreo & 5 & 1.745 & 0.124 \\
& Tipo de muestreo & 4 & 62.168 & 0.001 \\
& Época de muestreo * tipo de muestreo & 20 & 2.862 & 0.001 \\
\multirow{3}{*}{ Potrero } & 5 & 10.410 & 0.001 \\
& Época de muestreo & 3 & 25.721 & 0.001 \\
& Tipo de muestreo & 15 & 2.815 & 0.001 \\
\hline
\end{tabular}


En contraste con el hábitat bosque, en el potrero se encuentran diferencias significativas para cada variable y la interacción entre ellas.

Al realizar una prueba a posteriori Tukey para cada hábitat, en el bosque se encuentra que la variable tipo de muestreo presenta diferencias significativas ( $p$ : 0.05), agrupando a los muestreos cuadrante-manual y sacos mini-Winkler en un subgrupo, separándolos de cebo epigeo y trampa pitfall, siendo más eficiente el muestreo cuadrante-manual y sacos mini-Winkler.

En el potrero la variable época de muestreo presentó diferencias significativas ( $p$ : 0.05), presentándose tres subgrupos: el primero con la época uno representa el mes de julio, dos con las épocas 2, 5 y 6 corresponden a agosto, noviembre y diciembre y subgrupo 3 con épocas 3 y 4 correspondiente a septiembre y octubre. Así mismo, la variable tipo de muestreo presentó diferencias significativas ( $p$ : 0.05) en el potrero, agrupándolos en tres subgrupos, siendo más eficiente el muestreo manual seguido por las trampas pitfall y los sacos mini-Winkler registraron la menor captura.

\section{DISCUSIÓN}

\section{Composición general de la mirmecofauna}

El registro de las especies de hormigas para el relicto de bosque seco del JBC se encuentra por encima de lo reportado para otros relictos de bosque seco con área similar del Valle del Cauca: Colinder 12.3 ha con 37 especies y El Vínculo 15 ha con 41 especies, pero se encuentra por debajo de los bosques El Medio con 12.7 ha con 89 especies y Las Pilas 12.2 ha con 98 especies (Chacón de Ulloa \& Armbrecht, 2006). Myrmicinae, Ponerinae y Formicinae fueron las tres subfamilias dominantes en riqueza de especies, valores que coinciden con los registros para Colombia (Fernández, 2006).

La riqueza de hormigas encontrada, tanto en bosque como en potrero, es similar a lo registrado en estudios realizados por Armbrecht (1995);
Armbrecht \& Chacón de Ulloa (1997), donde se reportan entre el 85 y $93 \%$ de las especies de hormigas para los bosques y entre el 54 y $67 \%$ para las matrices (potreros ralos, potreros arbolados y cultivo de caña de azúcar). La intensificación de la producción agropecuaria, y en particular el establecimiento de potreros ralos, tienen un efecto sobre el suelo compactándolo y llevándolo a procesos erosivos; a su vez presionan la macrofauna de suelo y hojarasca. El bosque seco tropical ha sido reducido y aislado, de tal manera que cada vez es más vulnerable, como lo señala Arcila et al. (2012) en su estudio sobre el estado de fragmentación del bosque seco tropical en la cuenca alta del río Cauca. Sus resultados demuestran que los remanentes de este ecosistema tan solo representan el 1.8\% del área total (608 992 ha) y están distribuidos en más de 1600 fragmentos con tamaño promedio 6.03 ha.

La subfamilia Myrmicinae se encuentra en todos los trópicos del mundo y alcanza grandes números en áreas alteradas, ocupa una gran variedad de estratos de nidificación y presenta diversos hábitos alimenticios pasando por generalistas, cultivadoras y hasta carroñeras (Longino, 1994). Se destaca la presencia de la hormiga Wasmannia auropunctata conocida como hormiga vagabunda. Las especies de la subfamilia Ponerinae son característicamente cazadoras y sus nidos son muy frecuentes en madera descompuesta, hojarasca y suelo (Lattke, 2003), son consideradas benéficas al consumir otros artrópodos y servir de control biológico, además prefieren ecosistemas menos alterados y con mayor cobertura vegetal (Rivera \& Armbrecht, 2006).

\section{Diversidad de especies y estimadores de riqueza}

El valor del índice de Shannon sugiere que el bosque es un hábitat más diverso, lo cual se encuentra en concordancia con las características que presenta en cuanto a su estructura más compleja en comparación al potrero, un hábitat simplificado y desestructurado. 
Estos valores se hallan dentro de los rangos reportados en otros estudios en fragmentos de bosque seco y sus matrices (Armbrecht \& Chacón, 1997; 1999).

El valor calculado del índice de equitabilidad sugiere que las especies tienden a estar distribuidas equitativamente y con una ligera dominancia de algunas, en su orden Solenopsis sp. 1, Wasmannia auropunctata, Pheidole sp.4, y Gnamptogenys sp.1 que por su abundancia relativa se consideran dominan en el bosque. $W$. auropunctata es una especie frecuente en ambientes perturbados donde aprovechan el recurso e incrementan sus poblaciones (Achury et al., 2008).

En caso del potrero el valor del índice de dominancia indica la presencia de una especie dominante para este hábitat, en este caso la hormiga Solenopsis sp.2, sugiriendo una distribución menos equitativa de las especies.

La riqueza de especies exclusivas para bosque y potrero sugieren que el bosque preserva un mayor número de especies raras que la matriz, resultado importante de resaltar en la medida en que estos fragmentos de bosque requieren ser conservados para poder mantener esta fauna asociada. Resultados similares han sido encontrados en estudios realizados por Armbrecht \& Chacón (1997; 1999), donde los fragmentos de bosque seco relictuales estudiados en el Valle del Cauca aportan un número significativamente mayor de especies, en general las matrices no son de buena calidad y particularmente son dominadas por potreros ralos.

\section{Variables ambientales versus abundancia y riqueza de hormigas}

Se presentó una relación inversamente proporcional con esta variable ambiental en el bosque y directa en el potrero. Rivera \& Armbrecht (2006) reportaron que la temperatura del suelo es la variable abiótica que mejor explica la riqueza de especies y presentan una relación inversa (i.e. a medida que se incrementa la temperatura del sitio, el número de especies capturadas decrece). A pesar de que está reportado que las hormigas se encuentran limitadas a forrajear en temperaturas medias alrededor de los $30{ }^{\circ} \mathrm{C}$, debido a que son ectotermas y no resisten temperaturas ni muy bajas ni muy altas (Hölldobler \& Wilson, 1990), lo valores medios de temperatura del bs-T del Jardín Botánico fueron más elevados y siempre asociados con la presencia de hormigas.

La humedad es un factor microambiental que afecta, en conjunto con otros factores bióticos y abióticos, al número de especies encontradas. Para Schumacher \& Whitford (1976) la baja humedad influye desfavorablemente en el forrajeo. Este estudio fue consistente con dichos autores, puesto que a mayor humedad promedio se mantuvo una mayor riqueza de especies. Posiblemente al estar dentro del bosque, que es un hábitat que conserva por más tiempo la humedad cerca del suelo, más especies de hormigas pueden sobrevivir mejor que en la matriz de potrero.

\section{CONCLUSIONES}

El fragmento de Bosque Seco Tropical Jardín Botánico de Cali registra una mayor riqueza de especies de hormigas especialistas con respecto a la matriz de potrero en la cual está inmerso. A pesar de que el bosque se encuentra en un proceso de regeneración y que su matriz está fuertemente perturbada por constantes incendios e intervención humana, esta riqueza de especies de hormigas es una fuente de recursos biológicos importante y se constituye en una valiosa contribución a la conservación de la mirmecofauna del Valle del Cauca.

\section{AGRADECIMIENTOS}

Los autores expresan su agradecimiento a los trabajadores del Jardín Botánico de Cali por su colaboración y apoyo en el trabajo de campo, a la Universidad del Cauca y a la Universidad Autónoma de Occidente que financió el proyecto. A los revisores anónimos por sus valiosos comentarios y sugerencias para mejorar el manuscrito. 


\section{REFERENCIAS BIBLIOGRÁFICAS}

Achury, R., Chacón de Ulloa, P. \& Arcila, A. M. (2008). Composición de hormigas e interacciones competitivas con Wasmannia auropunctata en fragmentos de bosque seco tropical. Revista Colombiana de Entomología, 34, 209-216.

Aldana, R.C. \& Chacón de Ulloa, P.(1995). Hormigas (Hymenoptera: Formicidae) de la cuenca media del río Calima (Valle, Colombia). En Resúmenes Primera Reunión de la Sección Bolivariana. Unión Internacional para el estudio de los insectos sociales (IUSSI) (pp. 45 -46). Cali: Universidad del Valle.

Arcila, A.M., Valderrama, C., Chacón de Ulloa, P. (2012). Estado de fragmentación del bosque seco de la cuenca alta del río Cauca, Colombia. Biota Colombiana, 13(2), 86-100.

Armbrecht, I. (1995). Comparación de la mirmecofauna en fragmentos boscosos del Valle geográfico del río Cauca. Colombia. Boletín del Museo de Entomología de la Universidad del Valle, 3(2), 1-14.

Armbrecht, I. (1996). Análisis de la diversidad del ensamblaje de hormigas en fragmentos de bosque seco en el Valle del Cauca, Colombia. (Tesis de Magíster en Biología).Cali: Universidad del Valle. $120 p$.

Armbrecht, I. (1999). Rareza y Diversidad de Hormigas en Fragmentos de Bosque Seco Colombianos y sus Matrices. Biotrópica, 31(4), 646-653.

Armbrecht, I. \& Chacón de Ulloa, P. (1997). Composición y diversidad de hormigas en bosques secos relictuales y sus alrededores, en el Valle del Cauca, Colombia. Revista Colombiana de Entomología, 23(1-2), 45-50.

Armbrecht, I. \& Chacón de Ulloa, P. (1999). Rareza y diversidad de hormigas en fragmentos de bosque seco colombianos y sus matrices. Biotrópica, 31(4), 646-653.

Beattie, A. (1985). The evolutionary ecology of antplant mutulisms. UK -Cambridge: Cambridge University Press. 182 p.

Bestelmeyer, B. T., Agosti, D., Alonso, L. E., Brandão, C. R. F., Brown, W. L., Delabie, J. H. C. \& Silvestre, R. (2000). Field techniques for the study of ground-dwelling ant: an overview, description, and evaluation. En D. Agosti, Majer, J.D., Alonso, L. E. \& Schultz, T.R. (eds.). Ants: standard methods for measuring and monitoring biodiversity (280 pp). Washington, DC: Smithsonian Institution Press.

Boltón, B. (1994). Identification guide to the ant genera of the World. Cambrige Massachusetts: Harvard University press. $222 \mathrm{p}$.

Buckley, R. (1982) Ant-Plant interactions: A World Review. En R.C. Buckley (ed.). Ant-Plant interactions in Australia (pp 111-141). Queesland: Griffith University.

Bustos, J., \& Chacón de Ulloa, P. (1996). Mirmecofauna y perturbación en un bosque de niebla Neotropical (Reserva Natural Hato Viejo, Valle del Cauca, Colombia). Revista Biología Tropical, 4445, 259-266.

Colwell, R. K. (1998). User's guide to the richness estimator program, EstimateS. Versión 5.1. 13pp. Published at http://viceroy.eeb.uconn.edu/EstimateS

Colwell, R.K. \& Coddington, A. (1994). Estimating the extent of terrestrial biodiversity through extrapolation. Philosophical Transactions of the Royal Society London (Ser. B), 345, 101-118.

Corporación Autónoma Regional del Cauca-CVC- . (2000). Sistema de información geográfica de la unidad de manejo de cuenca Cali-Meléndez-Pance-Aguacatal. Santiago de Cali: Corporación Autónoma Regional del Cauca. 204 p.

Chacón de Ulloa, P. \& Armbrecht, I. (2006). Las hormigas del ecosistema bosque seco tropical. Tomo II. 345-351 p. En M.E. Chávez, \& Santamaría, M. (eds). Informe sobre el avance en el conocimiento y la información de biodiversidad 1998-2004. Bogotá D.C.: Instituto de Investigaciones de Recursos Biológicos Alexander Von Humboldt. Colombia.

Chacón de Ulloa, P., Osorio-García, A. M., Achury, R., \& Bermúdez-Rivas, C. (2012). Hormigas (Hymenoptera: Formicidae) del Bosque seco Tropical (bsT) de la cuenca alta del río Cauca, Colombia. Biota Colombiana, 13(2), 165-181.

Davidson, D., \& Mckey, D. (1993). The evolutionary ecology of simbiotic ant-plant relationships. Journal of Hymenoptera Research, 2(1), 18-33. 
Espinal, L.S. (1967). Apuntes sobre Ecología Colombiana. Cali: Universidad del Valle, Departamento de Biología. 32 p.

Fernández, F. (2003). Introducción a las hormigas de la región Neotropical. Bogotá: Instituto de Investigación de Recursos Biológicos Alexander von Humboldt.

Fernández, F. (2006). Caracterización de la diversidad de hormigas en Colombia. En M. E. Chaves, y Santamaría, M. (eds.). Informe sobre el avance en el conocimiento y la información de la biodiversidad 1998-2004. 2 Tomo (pp. 288-290). Bogotá: Instituto de Investigación de Recursos Biológicos Alexander von Humboldt. 429 p.

Fernández, F., \& Sendoya, S. (2004). List of Neotropical Ants (Hymenoptera: Formicidae). Revista Biota Colombiana, 5(1), 3-93.

Fundación Jardín Botánico de Cali. (2009). Primer informe Técnico y financiero del Proyecto "Diseño arquitectónico, paisajístico y ambiental del Ecoparque Río Cali". Cali: Fundación Jardín Botánico de Cali.

Holldobler, B., \& Wilson, E.O. (1990). The Ants. USA-Cambridge: Harvard University press. 732 p.

Instituto de Investigación de Recursos Biológicos Alexander Von Humboldt -IAvH-. (1998). Informe Nacional sobre el estado de la biodiversidad 1997- Colombia. 1998. Bosque seco tropical. En M.E. Chávez y Arango, N. (eds.). Tomo I : Diversidad Biológica(pp. 56-71). Bogotá: Instituto Humboldt, PNUMA, Ministerio del Medio Ambiente, vol. 3.

Jaffé, K., Lattke, J., \& Pérez., E.(1993). El mundo de las hormigas. Caracas: Equinoccio Ediciones-Universidad Simón Bolivar. 136p.

Kremen, C. (1994). Biological inventory using target taxa: a case study of the butterflies of Madagascar. Ecological Applications, 4, 407-422.

Lattke, J. E. (2003). Subfamilia Ponerinae. En F. Fernández (ed.). Introducción a las Hormigas de la
Región Neotropical(pp. xxvi+398). Bogotá: Instituto de Investigacion de Recursos Biológicos Alexander Von Humboldt,.

Longino, J. T. (1994). How to measure arthropod diversity in a tropical rainforest. Biology International, 28, 3-13.

McNelly, K., Miller, W., Reid, R., Mittermeier, R. \& Werner, T. (1990). Conserving the world's biological diversity. UICN, WRI, CI, WWF-US, World Bank. 193 p.

Moreno, C.F. (2001). Métodos para medir la biodiversidad. M\&T. Manuales \& Tesis SEA. Zaragoza: CYTED, ORCYT -UNESCO \& SEA (eds.). 84 p.

Palacio, E.E., \& Fernández, F. (2003). Clave para las subfamilias y géneros. En F. Fernández (ed.). Introducción a las Hormigas de la región Neotropical (XXVI + 398 p). Bogotá: Instituto de Investigación de Recursos Biológicos Alexander von Humboldt.

Orejuela, J. E. (2004). El Jardín Botánico de Cali: Un dinamizador del manejo ambiental y el desarroIlo social en la ciudad. El Hombre y la Máquina, 22, 1-8.

Orejuela, J. E. \& contreras, J.( 2006). The vegetation of the tropical dry forest of Cali Botanical Garden, Cali. Revista Lyonia, 9(2), 81-88.

Rivera, L. \& Armbrecht, I. (2006). Diversidad de tres gremios de hormigas en cafetales de sombra, de sol y bosques de Risaralda. Revista Colombiana de Entomología, 31(1), 84-96.

Roth, D. S., Perfecto, I., \& Bathcke, B. (1994). The effects of management systems on ground foraging ant diversity in Costa Rica. Ecological Applications, 4, 423-436.

Samways, M. (1983). Community structure of ants (Hymenoptera: Formicidae) in a series of habitats associated with citrus. Journal of Applied Ecology, 20, 833-847.

Schumacher, A., \& Whitford, W. G. (1976). Spatial and temporal variation in Chihuahuan desert ant faunas. Southwestern Naturalist, 21, 1-8.

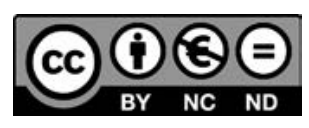

